\title{
Endoscopic Nd:YAG laser treatment of rectosigmoid
} cancer

\author{
L A Loizou, D Grigg, P B Boulos, S G Bown
}

\begin{abstract}
Forty nine patients with rectosigmoid carcinoma considered unsuitable for surgery underwent endoscopic Nd:YAG laser treatment for palliation of symptoms and tumour eradication, if feasible. Altogether $25(51 \%)$ of the lesions had distal margins less than $7 \mathrm{~cm}$ from the anus and $36(73 \%)$ extended above the peritoneal reflection. In seven patients with tumours less than $3 \mathrm{~cm}$ in diameter, symptomatic improvement was achieved in all (mean follow up 16 months) and complete tumour eradication in three. In the remaining 42 patients with larger tumours (34 greater than $2 / 3$ circumferential, mean length $5.5 \mathrm{~cm}$ ), symptomatic improvement was achieved with repeated treatments (average 3.4) in 31 (74\%) over a mean follow up of 19 weeks. Of the parameters assessed, only circumferential tumour extent proved significant in predicting functional outcome after treatment. All treatment failures (eight initial, three late) occurred in patients with extensive tumours, and only seven of these patients were considered fit for colostomy. Bowel perforation occurred in two patients $(5 \%)$ but there was no treatmentrelated mortality. Mean stay in hospital for all laser treatments was nine days $(30 \%$ were outpatient attendances). These results suggest that laser therapy may be the palliative treatment of choice in patients with rectal carcinoma unsuitable for surgery.
\end{abstract}

Statistics show that at the time of diagnosis approximately one third of rectosigmoid cancers are incurable because of advanced locoregional disease or distant metastases. ${ }^{12}$ The proper management of these patients remains unclear. Palliative resection has appreciable morbidity (18-50\%) and mortality (1-17\%), often requires the formation of a permanent colostomy, and involves a prolonged recovery period in patients with a limited survival. ${ }^{1-3}$ Defunctioning colostomy in patients with unresectable tumours, an operation with a mortality around $6 \%$, relieves obstructive symptoms but is of little value in palliating rectal bleeding, discharge, and tenesmus. ${ }^{3}$ Over the past 50 years several techniques have been used in an attempt to treat local symptoms in patients unsuitable for surgery because of high anaesthetic risk and as an alternative to palliative surgery in those with incurable disease. One of the more recent of these treatments is endoscopic neodymium: yttrium aluminium garnet (Nd:YAG) laser tumour photodestruction which was introduced in the early 1980s. Although several groups have reported a high initial success rate with laser treatment, very few have studied its longterm palliative efficacy. ${ }^{+8}$ The present study presents the initial and longterm results of palliative $\mathrm{Nd}$ :YAG laser therapy in 49 patients treated in our unit and attempts to analyse the importance of several patient and tumour parameters as determinants of functional outcome.

\section{Materials and methods}

\section{PATIENT POPULATION}

Forty nine patients with rectosigmoid adenocarcinomas received endoscopic laser therapy at the National Medical Laser Centre in the three year period 1985-8. They were referred because of incurable disease (documented distal metastases or advanced locoregional disease), high surgical risk (advanced age, severe cardiorespiratory disease), or refusal of surgery. Patients were divided into two groups according to tumour size. Those with tumours less than $3 \mathrm{~cm}$ in maximum diameter and less than $1 / 3$ circumferential were allocated to group 1; patients with larger lesions were allocated to group 2. Patient details, symptomatic presentation, and reasons for laser therapy in the two groups are shown in Table I. Three patients in group 1 had asymptomatic anastomotic recurrences after anterior resection of the rectum discovered at routine follow up. Twelve of the 16 patients with locally advanced disease in group 2 had symptomatic recurrences after anterior resection. Tumour details are shown in Table II. In

TABLE I Patient details, symptomatic presentation, and reasons for referral

\begin{tabular}{lcc}
\hline & $\begin{array}{c}\text { Group I } \\
(n=7)\end{array}$ & $\begin{array}{l}\text { Group 2 } \\
(n=42)\end{array}$ \\
\hline Sex ratio (M:F) & $4: 3$ & $20: 22$ \\
Mean (SD) age (yrs) & $71(14)$ & $73(12)$ \\
Symptomatic presentation: & & \\
$\quad$ Asymptomatic & 3 & 0 \\
RD/tenesmus/incontinence & 4 & 28 \\
$\quad$ Obstruction \pm RD & 0 & 14 \\
Reasons for laser treatment: & & \\
$\quad$ Liver metastases & 0 & 19 \\
$\quad$ Advanced local disease & 0 & 16 \\
$\quad$ High surgical risk/refusal of surgery & 7 & 7 \\
\hline
\end{tabular}

$\mathrm{RD}=$ rectal discharge

TABLE II Tumour details

\begin{tabular}{lll}
\hline & $\begin{array}{l}\text { Group } 1 \\
(n=7)\end{array}$ & $\begin{array}{l}\text { Group 2 } \\
(n=42)\end{array}$ \\
\hline Circumferential extent: & & \\
$\quad<1 / 3\left(C_{1}\right)$ & 7 & 0 \\
$1 / 3-2 / 3\left(C_{2}\right)$ & 0 & 8 \\
$>2 / 3\left(C_{3}\right)$ & 0 & 34 \\
Tumour length (mean (SD)) (cm) & $2 \cdot 0(0 \cdot 7)$ & $5 \cdot 5(1 \cdot 9)$ \\
Distal tumour margin $<7 \mathrm{~cm}$ from anus & 2 & 23 \\
Site: & 4 & 31 \\
$\quad$ Rectum & 3 & 11 \\
$\quad$ Rectosigmoid & 3 & \\
\hline
\end{tabular}

National Medical Laser
Centre and Department 26 September 198 
group 2 patients, eight tumours (19\%) were located totally below the peritoneal reflection of the rectum and $23(55 \%)$ had a distal margin less than $7 \mathrm{~cm}$ from the anal verge. If tumour resection had been attempted in the latter patients, most would have required an abdominoperineal operation with the fashioning of a permanent colostomy.

\section{ENDOSCOPIC TECHNIQUE}

Whenever possible treatments were performed on an outpatient basis. The rectum was prepared by the administration of sodium phosphate enemas and occasionally a washout. Many patients required no sedation or analgesia during the procedure but if necessary diazepam and pethidine were administered intravenously. Lesions encroaching onto the anal verge were treated under general anaesthesia as laser therapy at this site is exquisitely painful. A flexible sigmoidoscope (Fujinon S1G ET) with a safety filter in its eyepiece was used. The output of a continuous wave Nd:YAG laser (Flexilase, Living Technology, Glasgow), which can generate up to $100 \mathrm{~W}$ of radiation at a wavelength of $1064 \mathrm{~nm}$, was transmitted by a gas cooled (carbon dioxide or air) quartz fibre delivery system ( $1.8 \mathrm{~mm}$ outside diameter) which can be passed down the working channel of the sigmoidoscope. Insufflated gas and smoke produced during treatment was vented through the working channel by connecting it through its auxiliary inlet to a water-seal drain under a head of about $20 \mathrm{~cm}$ of water which maintained adequate luminal distention. The aim of treatment was to destroy as much tumour as possible in one session. The fibre tip was positioned 5-10 $\mathrm{mm}$ from the target tissue and exophytic tumour nodules were shaved back close to the rectosigmoid wall by vaporisation using high power pulses (60-80 W, 1-2 second shots). The last 3-4 mm of luminal tumour and flat sessile lesions were treated by coagulation alone (50-60 W, 1 second shots) in order to minimise the risk of perforation.

Whenever possible treatment of tumours causing significant luminal narrowing began at their superior margin and proceeded inferiorly, as immediate oedema in unvaporised areas can hinder progress in the forward direction and make visualisation difficult. For tumours impassable with the sigmoidoscope, dilatation with through-scope balloon dilators was attempted in order to provide access to their superior margin, although this often proved unsuccessful. Inadequately dilated and completely obstructing tumours were treated in a forward direction, recanalising $1-2 \mathrm{~cm}$ of tumour at any one session. Treatments were repeated at intervals of three to seven days, allowing time for sloughing of necrosed tumour, until adequate tumour destruction was achieved. After successful initial laser therapy, treatments were repeated electively at intervals of six to eight weeks. This time interval was chosen as our previous experience and that of others, indicated that appreciable tumour regrowth causing recurrent symptoms occurred in most patients at about this time. ${ }^{+8}$ In addition, it seems that the longterm efficacy of laser therapy is less if patients are retreated at longer time intervals after the development of recurrent symptoms, ${ }^{9}$ as discussed below.

\section{PATIENT EVALUATION}

Group 1. In asymptomatic patients, treatment was considered successful if complete macroand microscopic eradication of the tumour was achieved. In those presenting with rectal discharge or tenesmus, treatment was deemed successful if they were rendered completely asymptomatic.

Group 2. Evaluation of the effect of treatment in this group of patients is more difficult and to a great extent subjective, especially in those presenting with rectal discharge, haemorrhage, incontinence, and tenesmus. Pain caused by local pelvic infiltration cannot be palliated by laser therapy and has not been assessed. In a previous report, ${ }^{+}$we attempted to quantify objectively these symptoms, and others have done likewise. ${ }^{89}$ Our subsequent experience, however, has shown that this is extremely difficult to perform especially in elderly, terminally ill patients who are seldom able to give a reliable objective account of their symptoms. For the patients described in this report we have used a more subjective assessment - laser therapy was considered successful if symptoms caused by intraluminal tumour were reduced to an extent that enabled the patient to lead a lifestyle appropriate for his or her age and general condition without constant concern for bowel function. Thus treatment success depended on both the patient's subjective symptomatic assessment and attitude towards these symptoms. In patients with predominantly obstructive symptoms, clinical examination findings and the degree of colonic faecal loading on a plain abdominal radiograph were used in addition to subjective symptomatic assessment to assess treatment success.

\section{STATISTICS}

Statistical analyses were performed using a $\chi^{2}$ test with Yates's correction and Mann-Whitney U test. Survival and symptom palliation interval distributions were calculated using the life table method and tested for disparity by log rank analysis. For the calculation of these distributions, patients dying palliated were censored (that is, lost to follow up)

\section{Results}

GROUP 1

Complete resolution of symptoms was achieved in all four patients presenting with rectal discharge with or without tenesmus and was maintained until death, which occurred from unrelated disease at $13,15,16$, and 20 months respectively after entry into the study. Complete macroscopic and microscopic tumour eradication was possible in only one of these four patients

In the three asymptomatic patients, complete tumour destruction with negative biopsy specimens was achieved in two. The patient with 
Survival and symptom palliation interval (SPI) distributions calculated by the life table method for patients with advanced rectosigmoid cancers (group

2). The distributions are significantly disparate $(p<0.005)$ by log rank $(p<0.005)$ by $\log$ rank
analysis. At the time of writing, only seven patients are still alive at a mean follow up of 5 months (range 2-13 months).

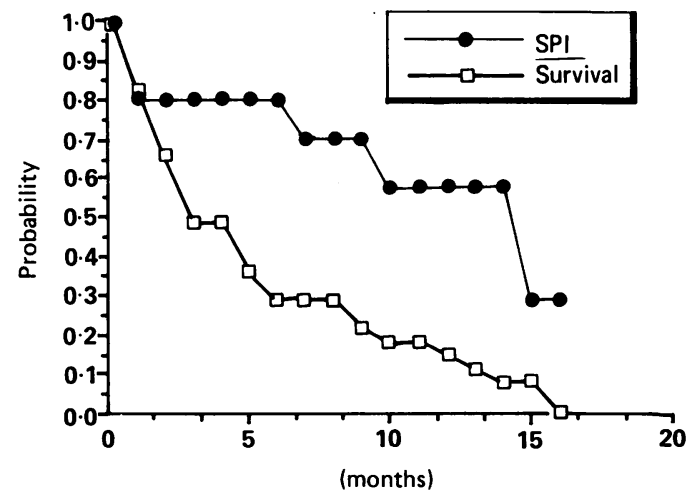

residual microscopic adenocarcinoma received a $60 \mathrm{~Gy}$ course of external beam radiotherapy with complete tumour eradication. All three patients are alive and free of tumour at 12, 20, and 28 months respectively. The average number of laser treatments required to eradicate macroscopic tumour was $3 \cdot 5$ (range $2-5$ ).

\section{GROUP 2}

Symptomatic palliation. Laser therapy was initially successful in 34 patients $(81 \%)$; symptomatic improvement was maintained by repeated treatments in 31 patients $(74 \%)$ over a mean follow up period of 4.8 months (range 1-16 months). At the time of writing only seven patients are still alive at a mean follow up of five months (range 2-13). Survival and symptom palliation interval distributions calculated using the life table method are shown in the Figure. Median survival was only four months with a $15 \%$ one-year survival. The survival and symptom palliation interval distributions are significantly disparate by $\log$ rank analysis $(\mathrm{p}<0.005)$ indicating that most patients are dying symptomatically palliated.

Symptomatic palliation rates analysed according to circumferential tumour extent are shown in Table III. The initial success of laser therapy was significantly greater in patients with $\mathrm{C}_{2}$ than $\mathrm{C}_{3}$ tumours $(\mathrm{p}<0 \cdot 01)$. Although the longterm success rate was numerically greater in patients with $\mathrm{C}_{3}$ tumours, the difference did not reach statistical significance. The follow up of the latter, however, was shorter than that of patients

TABLE III Functional outcome after laser therapy according to circumferential tumour extent $\left(C_{2}: 1 / 3-2 / 3, C_{3}:>2 / 3\right)$

\begin{tabular}{lll}
\hline & $\begin{array}{l}\text { Early functional } \\
\text { success } \\
\text { No } \text { grade }\end{array}$ & $\begin{array}{l}\text { Longterm functional } \\
\text { success } \\
\text { No }(\%)\end{array}$ \\
\hline $\mathrm{C}_{2}(\mathrm{n}=8)$ & $8(100)^{\star}$ & $7(88)$ \\
$\mathrm{C}_{3}(\mathrm{n}=34)$ & $26(76)^{\star}$ & $24(71)$ \\
\hline${ }^{\star} \mathrm{p}<0.01$. & &
\end{tabular}

with $\mathrm{C}_{2}$ tumours $(4 \cdot 25$ v 6.5 months $)$. In contrast to circumferential tumour extent, tumour length and location (rectum or rectosigmoid region) had no prognostic importance as indicators of successful initial outcome after laser treatment. In group 2, the initial and longterm success of laser therapy in patients with anastomotic recurrences and primary tumours were very similar.

The functional outcome of patients analysed according to the predominant presenting symptom and the management of treatment failures are shown in Table IV. Both the early and longterm success rates of laser therapy were numerically but not significantly greater in patients with rectal discharge, incontinence, and tenesmus than in those with obstructive symptoms. It should be noted that after successful initial treatment symptomatic improvement could be maintained long term by repeat laser sessions in $91 \%$ of patients. Two thirds of the patients in whom treatment failed were managed by defunctioning colostomy, the remaining patients were considered too unwell for further intervention. In the group of patients presenting with rectal discharge, treatment failures occurred because of anal incontinence despite adequate tumour destruction, presumably secondary to tumour involvement of the anal sphincter mechanism. In patients with obstructive symptoms one early and one late failure were due to laser induced perforation and these patients both were managed by defunctioning colostomy.

Treatment requirements and admission to hospital. Initial symptomatic improvement occurred after an average of 1.3 weeks and a mean of 1.6 treatments were required. On average, patients underwent a total of 3.4 procedures in order to maintain this improvement during their survival. A mean of $8000 \mathrm{~J}$ of $\mathrm{Nd}$ :YAG laser energy was delivered per procedure. Some $30 \%$ of laser treatments were given on an outpatient basis. The average stay in hospital, excluding terminal care, was nine days (each day case attendance was counted as one day in hospital).

Treatment complications. Procedures were generally well tolerated; some patients experienced transient abdominal distension and discomfort as a result of the gas insufflated during treatment. Laser induced perforation occurred in two patients $(5 \%)$ with impassable circumferential carcinomas at the rectosigmoid junction: both were managed by defunctioning colostomy. One patient developed localised peritonism and transient pyrexia within 12 hours of laser therapy; a gastrograffin enema did not detect a leak and the patient made an uneventful recovery on conservative management. There was no procedurerelated mortality in this series.

TABLE IV Functional outcome after laser therapy according to main presenting symptoms and management of treatment failures

\begin{tabular}{|c|c|c|c|}
\hline Presenting symptom & $\begin{array}{l}\text { Early functional } \\
\text { success } \\
\text { No }(\%)\end{array}$ & $\begin{array}{l}\text { Longterm functional } \\
\text { success } \\
\text { No }(\%)\end{array}$ & Management of failures \\
\hline Rectal discharge/incontinence $(n=27)$ & $23(85)$ & $21(78)$ & $\begin{array}{l}\text { Early }\left\{\begin{array}{l}\text { Defunctioning colostomy (2) } \\
\text { Terminal care (2) }\end{array}\right. \\
\text { Late } \\
\text { Defunctioning colostomy (2) }\end{array}$ \\
\hline Obstruction $(n=15)$ & $11(69)$ & $10(67)$ & $\begin{array}{l}\text { Early }\left\{\begin{array}{l}\text { Defunctioning colostomy (2) } \\
\text { Terminal care }(2)\end{array}\right. \\
\text { Late }\end{array}$ \\
\hline
\end{tabular}


Survival. The mean (SD) survival of group 2 patients was $4.6(4.0)$ months. Survival probability decreased rapidly $(0.65,0.50,0 \cdot 30,0 \cdot 15$ at $2,4,8,12$ months respectively) with no survivors after 16 months (Figure). There was no significant difference in mean survival when patients were stratified according to symptomatic presentation (rectal discharge with or without tenesmus: $4 \cdot 7$, obstruction: 4.5 months), circumferential tumour extent $\left(\mathrm{C}_{2}\right.$ : $6 \cdot 4, C_{3}: 4.2$ months) and the presence or absence of hepatic metastases $(4.4$ and 4.8 months respectively)

\section{Discussion}

The aims of any palliative treatment for rectosigmoid cancer are to improve local symptoms and the patients' quality of life with minimal morbidity and mortality. Nd:YAG laser therapy in patients with advanced lesions (group 2) in this study provided successful initial palliation in $81 \%$. Our experience is in agreement with that of other groups who have reported initial success rates in similar patients of $80-90 \% .^{6-9}$ Importantly, symptomatic improvement occurs early without the need for prolonged stay in hospital. An average of 1.6 treatments given over 1.3 weeks in our study and 2.5 treatments over 2.4 weeks in Brunetaud et al's study ${ }^{8}$ were necessary to achieve initial symptomatic improvement. Symptomatic palliation was maintained by repeated treatments in $74 \%$ of our group 2 patients over a mean follow up period of 19 weeks. Brunetaud et al's experience is similar; the proportions of surviving patients remaining palliated at $6,9,12$, and 24 months were $90 \%$, $89 \%, 82 \%$, and $64 \%$ respectively. In contrast, a recent study from Belgium reported much lower symptomatic improvement in surviving patients $-51 \%, 41 \%$, and $25 \%$ at 6,12 , and 18 months respectively. ${ }^{9}$ The rather poor longterm outcome was attributed by the authors to progressive growth of the extrinsic component of the tumour which is not amenable to laser therapy. It should be noted, however, that patients in the Belgian study were often not retreated electively but only when they developed recurrent symptoms with an average treatment interval of $4 \cdot 7$ months, which is significantly longer than in our study ( 2.6 months). With longer intervals between repeat sessions, endoscopic treatments become technically more difficult because of the larger tumour bulk, and the chances of maintaining palliation are reduced.

In order to identify prognostic indicators of initial and longterm functional outcome after laser therapy in group 2 patients, we examined the importance of the following patient and tumour characteristics: reason for laser treatment (hepatic metastases, advanced local disease, recurrence after surgery, high surgical risk), symptomatic presentation (rectal discharge with or without incontinence or obstructive symptoms predominant), tumour location (rectum or rectosigmoid junction), and circumferential tumour extent $\left(\mathrm{C}_{2}\right.$ or $\left.\mathrm{C}_{3}\right)$. Only the latter proved to be of any significance; initial success was significantly higher in $\mathrm{C}_{2}$ tumours but the improved outcome was not maintained long term. Our results are in exact agreement with those of Brunetaud et al who have a more extensive experience. ${ }^{10}$ In contrast, Van Custem et al have found the $\mathrm{C}$ grade of the tumour to be of no prognostic significance. In their study longterm functional outcome was significantly worse in patients with recurrent carcinoma after previous resection, presumably because of extensive extraluminal disease, locally advanced tumours (high T grade), and lesions greater than $4 \mathrm{~cm}$ in length. ${ }^{9}$

At least $60 \%$ of group 2 patients in our study would have required a permanent colostomy had they been managed surgically and laser therapy avoided the need for this in $74 \%$ of these patients. Two thirds of the treatment failures in the subgroup with discharge and anal incontinence at presentation were functional with persistent symptoms despite adequate tumour ablation. All these patients had low rectal tumours and tumour invasion of the anal sphincter mechanism may have been responsible for the lack of improvement. The remaining failures in this subgroup occurred in patients with massive tumours at presentation. Treatment failures in patients presenting with obstructive symptoms occurred because of inadequate recanalisation $(60 \%)$ or perforation $(40 \%)$ of completely circumferential tumours. It should be noted that $50 \%$ of initial laser treatment failures were in patients considered too unwell for any further intervention.

A palliative treatment will only become accepted if its therapeutic efficacy is matched by a low morbidity and mortality, it is well tolerated by patients, and requires only a short stay in hospital. Laser therapy for advanced rectal cancers fulfills all these criteria. There was a 5\% perforation rate, $2 \%$ minor complication rate, and nil procedure related mortality in this series. Other groups have reported major (perforation, fistulation, sepsis, and bleeding) and minor complications (transient pyrexia and bleeding not requiring transfusion) in $3-10 \%$ and $5-10 \%$ of patients respectively and a mortality of $1-2.5 \% .^{689}$ Laser therapy is generally well tolerated; patients experience minor discomfort during treatment due to a local heating effect and may have increased rectal discharge for 24-48 hours afterwards due to sloughing of necrosed tumour. Importantly, because of the lack of requirement for general anaesthesia and full bowel preparation, treatments can be performed on a day-case basis in ambulant patients thus minimising time in hospital. All these factors should contribute towards improving the quality of life of these unfortunate patients; this has now been documented in a prospective assessment of quality of life using two different instruments in a group of 14 patients with advanced rectal cancer."

How does endoscopic laser therapy compare with other non-surgical palliative treatments for rectosigmoid cancer? Although external beam and afterloading radiotherapy have produced excellent results in small rectosigmoid tumours, palliation of more advanced lesions is much less satisfactory. Rectal discharge and tenesmus are improved in approximately $50 \%$ but in patients with obstructing tumours a defunctioning colos- 
tomy is often necessary during the course of treatment. The symptomatic benefit from radiotherapy is often shortlived (less than three months) and radiation proctitis, delayed haemorrhage, and fistulation occur commonly. ${ }^{12-15}$ Adjuvant radiotherapy after laser treatment should theoretically be of benefit by reducing intraluminal tumour regrowth and hence the frequency of repeat laser sessions. In addition, shrinkage of the extrinsic tumour component should prevent or delay luminal compression and hopefully reduce pain due to pelvic infiltration. Like laser therapy, cryosurgery is easy to perform, requires no anaesthesia except for very low tumours, and is well tolerated but repeated applications are necessary to maintain symptomatic improvement. It is, however, limited to tumours below the peritoneal reflection of the rectum because of the risk of perforation. Good tumour debulking can be achieved but tumour sloughing and rectal discharge may continue for up to two weeks. ${ }^{16}$ The efficacy of cryosurgery is very similar to that of the laser. In a recent study, longterm palliation of rectal discharge and tenesmus was possible in $65 \%$ of patients, and colostomy was avoided until death (mean survival 2.3 years) in $78 \%$. There was only a $5 \%$ perforation and mortality rate in this series but other serious complications occurred in $34 \%$ of patients. ${ }^{17}$ Electrocoagulation has a high success rate (77-94\%) but requires general or regional anaesthesia and cannot be used above the peritoneal reflection of the rectum. Like cryosurgery, electrocoagulation has a high complication rate (up to $30 \%$ ) reflecting the lack of treatment precision and unpredictable extent of tissue effects with these methods. ${ }^{18-20}$

Our results, and the experience of others, indicate that in small rectal carcinomas laser therapy can achieve excellent symptomatic palliation and complete macroscopic and microscopic eradication of tumour in up to $100 \%$ of patients, with minimal associated morbidity. ${ }^{78}$ Electrocoagulation, cryosurgery, and brachytherapy are just as effective as laser therapy in the treatment of small lesions but their limitations and complications are the same as for the treatment of more extensive tumours detailed above. ${ }^{13-20}$ Despite such good results, curative local treatment of small rectal cancers with laser photoablation remains controversial. At presentation, $7-12 \%$ of carcinomas affecting the submucosa and muscularis propria of the rectum (UICC stage $\mathrm{T}_{2}$ ) have already metastasised to local lymph nodes and are therefore beyond the scope of local treatment. ${ }^{21}$ Endoscopic ultrasound is at present the most sensitive means of assessing lymph node status but its accuracy is still no greater than $80 \% .{ }^{22}$ In operable patients with early rectal cancers radical surgery remains, therefore, the treatment of choice. Curative laser therapy is justified, however, in those refusing surgery and in elderly and poor surgical risk patients, especially when abdominoperineal resection is the alternative.

Endoscopic laser photoablation should be considered as the palliative treatment of choice in patients with rectal carcinoma not suitable for surgery. It is applicable to lesions both above and below the peritoneal reflection and provides good longterm symptomatic control in a high percentage of patients, with a low morbidity and mortality unparalleled by any of the rival treatment methods. Laser therapy could also be used as an alternative to palliative surgery in some patients with incurable disease given the significant morbidity and mortality of the latter. As complete eradication of small tumours (less than $3 \mathrm{~cm}$ diameter) is possible in virtually $100 \%$ of patients with these, curative local laser treatment should be considered in highly selected patients.

1 Longo WE, Ballantyne GH, Bilchik AJ, Modlin IM. Advanced rectal cancer - what is the best palliation? Dis $\mathrm{Co}$ Rect 1988; 31: 842-7.

2 Bordos DC, Baker RR, Cameron JL. An evaluation of palliative abdominoperineal resection for carcinoma of the palliative abdominoperineal resection for carcind

3 Moran MR, Rothenberger DA, Lahr CJ, Buls JG, Goldberg SM. Palliation for rectal cancer - resection? Anastomosis? Arch Surg 1987; 122: 640-3.

4 Bown SG, Barr H, Matthewson K, et al. Endoscopic treatment of inoperable colorectal cancers with the Neodymium-YAG laser. Br $\mathcal{F}$ Surg 1986; 73: 949-52.

5 Naveau S, Roulot D, Zourabinchvili O, Poitrine A, Poynard $T$, Chaput JC. Traitement palliatif des adenocarcinomes du rectum par le laser YAG Neodyme: Resultats immediats et a long terme. Gastroenterol Clin Biol 1986; 10: 651-5.

6 Mathus-Vliegen EM, Tytgat GN. Nd:YAG laser photocoagulation in gastroenterology: its role in palliation of colorectal ulation in gastroenterology: its role in
cancer. Lasers Med Sci 1986: 1: 75-80.

7 Escourou J, Delvaux M, Frexinos J, et al. Traitement du cancer du rectum par le laser neodyme YAG. Gastroenterol Clin Biol 1986; 10: 152-7.

8 Brunetaud JM, Manoury V, Durcotte P, Cochelard D, Corto A, Paris JC. Palliative treatment of rectosigmoid carcinoma by laser endoscopic photoablation. Gastroenterology 1987; 92: $663-8$

9 Van Custem E, Boonen A, Geboes G, et al. Risk factors which determine long term outcome of Neodymium-YAG lase palliation of colorectal carcinoma. Int $\mathcal{f}$ Colorectal Dis 1989 4: 9-11.

10. Brunetaud JM, Maunoury V, Cochelard D, Boniface B, Cortot A, Paris JC. Parameters affecting laser palliation in patients with advanced digestive cancers. Lasers Surg Med 1989; 9: 169-73.

11 McGowan I, Barr H, Krasner N. Palliative laser therapy for inoperable rectal cancer. Does it work? A prospective study of quality of life. Cancer 1989; 63: 967-9.

12 Williams IG, Shulman IM, Todd IP. The treatment of recurrent carcinoma of the rectum by supervoltage $x$-ray therapy. Brf Surg 1956; 44: 506-8.

13 Papillon J. New prospects in the conservative treatment of rectal cancer. Dis Colon Rectum 1984; 27: 695-700.

14 Sichy B, Remington JH, Sobel SH. Treatment of recta carcinoma by means of endocavitary irradiation: a progres report. Cancer 1980; 46: 1957-61.

15 Puthawala AA, Syded AMN, Gates TC, McNamara C. Definitive treatment of extensive anorectal carcinoma by external and interstitial irradiation. Cancer 1982; 50: 1746-50.

16 Mlasowsky B, Duben W, Jung D. Crysosurgery for palliation of rectal tumours. F Exp Clin Cancer Res 1985; 4: 81-3.

17 Heberer G, Denecke H, Demmel N, Wirsching R. Loca procedures in the management of rectal cancer. World $\mathcal{F}$ Surg 1987; 11 : 499-503.

18 Hughes EP, Veidenheimer MC, Corman ML, et al. Electrocoagulation of rectal cancer. Dis Colon Rectum 1982; 25 215-8.

19 Madden JL, Kandalaft SI. Electrocoagulation as a primary curative method in the treatment of carcinoma of the rectum. Surg Gynecol Obstet 1983; 157: 164-79.

20 Hoekstra HJ, Verschuren RC, Oldhoff J, et al. Palliative and curative electrocoagulation for rectal cancer: experience and results. Cancer 1985; 55:210-13.

21 Goldberg SM, Thorson AG. Local therapy for early malignan lesions in the rectum. Eur f Surg Oncol 1987; 13: 155-7.

2 Beynon J, McC. Mortensen NJ. Staging of rectal cancer with endosonography. Hosp Update 1988; 14: 1748-54. 